\title{
Ecological Economics: Eine Leerstelle in den Lehrbüchern der Umweltökonomie
}

Aus Anlaß des Themenschwerpunktes Ecological Exonomics soll an dieser Stelle ein Blick in einige deutschsprachige Lehrbücher zur Umweltökonomie (siehe Kasten) geworfen werden. Leitendes Interesse dabei ist, ob und inwieweit Spuren der ökologischen Ökonomie in diesen Büchern zu finden sind. Es geht dabei nicht darum, die Bücher nach ihrer Art und Weise der jeweiligen Darstellung herkömmlicher Umweltökonomie zu beurteilen, sondern darum, wieweit Konzepte und Ansätze der ökologischen Ökonomie Berücksichtigung finden.

Als Richtschnur soll zum einen die Unterteilung in Scale (Ausmaß), Allokation und Verteilung gewählt werden, die sich innnerhalb der ökologischen Ökonomie als eine Art »Standard « zur Beschreibung der Aufgabenfelder durchgesetzt hat. Zum anderen soll das Buch von Pearce und Turner 1990 als »Referenz « herangezogen werden, da es zumindest als ein Wegbereiter der ökologischen Ökonomie angesehen werden kann und von dem schon mehrere Neuauflagen erschienen sind.

\section{Voranalytische Visionen}

Das einzige deutschsprachige Lehrbuch, das auf den Ansatz der ökologischen Ökonomie etwas eingeht, ist das von Cansier. Nimmt man die oben genannte Unterteilung, dann bleiben die übrigen deutschsprachigen Lehrbücher fast vollständig im Bereich der Allokationstheorie: Das Umweltproblem wird als eines verstanden, das auf die ineffiziente Allokation von Umweltgütern zurückzuführen ist. Dies bedeutet, daß die »... gefällte Entscheidung zugunsten einer wohlfahrtstheoretischen Methodik impliziert, daß dann, wenn Umweltgüter in diesem Sinne effizient alloziiert wären, aus ökonomischer Sicht kein Umweltproblem existieren würde« (Weimann: 29). Oder: »Folgerichtig muß die Aufgabe bei Umweltgütern - wie bei allen anderen Gütern auch - darin gesehen werden, diese so zu alloziieren, daß die Wohlfahrt der Volkswirtschaft maximiert wird « (Feess: 1). Und auch Endres sieht in der Internalisierung externer Effekte und dem Erreichen optimaler Umweltqualitätsniveaus - wenn auch letztlich nur als Referenzpunkt - den Beitrag der Umweltökonomie (Endres: 27),

$\mathrm{Ob}$ aber die externen Effekte als analytischer $\mathrm{Zu}$ gang zum Umweltproblem überhaupt adäquat sind, wird damit nicht mehr gefragt. Oder ob Umweltgüter tatsächlich Güter wie alle anderen Güter auch sind? Ob die mit der Wohlfahrtstheorie verbundene Annahme der Substitutionalität angemessen ist, wird nicht in Frage gestellt. Und die Problematik, die sich daraus ergibt, daß zukünftig Lebende nicht auf heutigen Märkten ihre Präferenzen äußern können, tritt in den Hintergrund. Die Wechselbeziehung zwischen den
Systemen Ökonomie und "Ökologie« bleibt ebenfalls unbetrachtet.

Das die effiziente Allokation von Umweltgütern auch innerhalb der ökologischen Ökonomie eine bedeutende Rolle spielt, steht ja außer Zweifel (siehe z. B. Folke, Costanza [1994]: Ecological Economics and Sustainable Development, Paper prepared for the Experts Meeting for the Operationalization of the Economics of Sustainability). Angesichts von Problemen wie dem Bevölkerungswachstum wird eine effiziente Allokation

- Dieter Cansier: Umweltökonomie. Gustav Fischer, Stuttgart 1993. 396 S., DM 49,80

- Alfred Endres: Umweltökonomie. Eine Einführung. Wiss. Buchgesellschaft, Darmstadt 1994. 191 S., DM

- Eberhard Feess: Umweltökonomie und Umweltpolitik. Vahlen, München 1995. 249 S., DM 38,-

- David W. Pearce, R. Kerry Turner 1990: Economics of Natural Resources and the Environment. Harvester Wheatsheaf, New York u. a., $378 \mathrm{~S}$.

- Joachim Weimann: Umweltökonomik. Eine theorieorientierte Einführung. Springer, Berlin u. a. 1995, 3. Aufl. 325 S., DM 45,-

sogar als eine wesentliche Aufgabe angesehen, um die entstehenden Verteilungskonflikte so klein wie möglich zu halten. Doch solche Probleme werden in Vorworten oder einleitenden Grundlagenkapiteln behandelt, die dem eigenlichen ökonomischen Teil vorangestellt sind. In diesem folgt dann weitgehend das Standardprogramm der neoklassischen Umweltökonomie (Internalisierung nach Coase und Pigou, Diskussion der Instrumente), das allerdings durch sehr interessante Ausführungen wie z. B. von Weimann und auch Feess zur Spieltheorie bereichert wird.

Cansier geht über diese Form der voranalytischen Vision deutlich hinaus: In den beiden Kapiteln »Abhängigkeit der Ökonomie von der Natur « und »Die anzustrebende Umweltqualität « geht er explizit auf das Abhängigkeitsverhältnis der Ökonomie von der Natur ein, indem er die Ökonomie als Durchflußsystem beschreibt und physikalische Restriktionen in Form der Hauptsätze der Thermodynamik beschreibt (Probleme des Scale). Und im Kapitel über die Umweltqualität diskutiert er u. a. Konzepte wie das des konstanten Umweltkapitalstocks im Rahmen einer nachhaltigen Entwicklung und einer ökologisch orientierten präventiven Politik, dem ökonomische Kosten-Nutzen-Abwägungen allein zu unsicher sind. Damit kommt er der Vorgehensweise von Pearce und Turner sehr nahe, die zu Anfang ihres Buches u. a. auf die Interaktion zwischen Umwelt und Ökonomie eingehen und in einem gesonder- ten Kapitel eine »Sustainable Economy« behandeln (Chapter 3).

Allerdings bleiben diese beiden Kapitel bei Cansier im wesentlichen unverbunden mit dem dann wieder konventionellen Teil über die neoklassische Umweltökonomie. So geht er im Bereich der ökonomischen Bewertungsverfahren dann nicht auf »Instrumente « wie den Safe-MinimumStandard ein, der dem Markt als Allokationsmechanismus Grenzen setzt und im Bereich der Naturschutz-Ökonomie eine bedeutende Rolle spielt. Solcher Art Verbindungen sind dagegen in dem Buch von Pearce und Turner häufig; das Buch hinterläßt nicht den Eindruck, daß die allokationstheoretisch fundierte Umweltökonomie und die Grenzen dieser Vorgehensweise getrennt voneinander behandelt werden. Anders gesagt: Nicht nur der Bereich der Allokation, sondern auch die Fragen der intra- und intergenerativen Verteilung und des Scale (Ausmaßes) finden explizit Berücksichtigung.

Begrenzungen der traditionellen Umweltökonomie treten in den hier aufgenommen deutschsprachigen Büchern dann meist im Zusammenhang mit der Instrumentendiskussion auf. Dies aber im wesentlichen nicht aufgrund der Einsicht in das Wechselverhältnis zwischen Ökonomie und Ökologie, sondern aufgrund der Probleme, die bei der Implementation von Pareto-effizienten Lösungen auftreten. Die Frage der ökologischen Standards wird quasi »externalisiert« an die Politik, die diese zu setzen hat und sich dann der Instrumente bedient, um die Standards kosteneffizient umzusetzen. Die Frage der Ökologie reduziert sich häufig auf die Angemessenheit der ökonomischen Instrumente und das Kriterium der ökologischen Treffsicherheit. Ausführungen wie der Exkurs bei Weimann über »Emissionen-Diffusionen-Immissionen « werden aber nicht mit dem Ansatz der Umweltökonomie rückgekoppelt.

Um nicht falsch verstanden zu werden: Die Kritik richtet sich nicht gegen eine allokationstheoretische Behandlung der Umweltprobleme, sondern gegen ihre fast ausschließliche Thematisierung. Auch wenn die jeweiligen Autoren Vorbehalte gegenüber der ökologischen Ökonomie haben mögen, die sicher in einigen Feldern begründet sind, so ist dies kein Grund, sie nicht wenigsten zu diskutieren und die für sie bestehenden Vorteile der neoklassischen Umweltökonomie gegenüber der ökologischen Ökonomie darzustellen. Dies wäre gerade für Lehrbücher eine wichtige Aufgabe.

\section{Eine Empfehlung}

Eine Empfehlung an Studierende und Lehrende aufgrund dieses an einigen Stellen sicher zu holzschnittartigen Vergleichs würde aus meiner Sicht wie folgt aussehen: Als Standardtext sollte auf das Buch von Pearce und Turner zurückgegriffen werden. Es bietet nicht nur einen guten Überblick über die neoklassische Umweltökonomie, sondern erweitert auch das Feld in Richtung der ökologischen Ökonomie und verknüpft 
beide miteinander. Für »Spezialthemen « wäre dann jeweils auf eines der übrigen Bücher zurückzugreifen: So sind die Ausführungen von Weimann zu Dilemmata und den Ergebnissen der Spieltheorie auf jeden Fall sehr lesenswert. Und einen Éinstieg in makroökonomische Aspekte von Umweltfragen findet sich bei Cansier, während das Buch von Fees ein längeres Kapitel über internationale Aspekte des Umweltproblems enthält.

Ansonsten ist zu hoffen, daß irgendwo an einem Schreibtisch schon jemand sitzt, um ein neues
Lehrbuch für den deutschsprachigen Raum über die ökologische Ökonomie zu schreiben. Einen Käufer kann ich Autor und Verlag schon jetzt garantieren!

Jürgen Meyerhoff, Berlin

\section{In memoriam Nicholas Georgescu-Roegen}

Im November dieses Jahres jährt sich zum ersten Mal der Todestag von Nicolas Georgesu-Roegen, ein gebührender Anlaß, seiner - neben etwa Kenneth Boulding - als einem der wenigen maßgeblichen Wegbereiter der ökologischen Ökonomie zu gedenken.

Georgescu-Roegens Laufbahn umschließt drei wichtige Wendepunkte in seinem Leben. Er begann als reiner Mathematiker und wurde Statistiker, dann mathematischer Ökonom und schließlich einer der tiefgründigsten und prominentesten Dissidenten der Wirtschaftswissenschaften. Wiewohl er in keinster Weise ein Außenseiter ist, hat sich kein renommierter Ökonom mit seiner Arbeit öffentlich auseinandergesetzt; stattdessen ist sie von den Standard-Ökonomen weitestgehend übergangen worden - ein freilich nicht untypisches Schicksal für Dissidenten. Jedoch ist die der Förderung des bioökonomischen Paradigmas gewidmete »European Association for Bioeconomic Studies « (EABS) ebenso ein Ausdruck für das Interesse an seiner Arbeit wie die Übersetzungen seines Hauptwerkes in andere Sprachen als Klassiker der ökologisch orientierten Ökonomik.

Georgescu-Roegen wurde am 4. Februar 1906 in Constanza, Rumänien, geboren. Sein Vater, ein Kapitän der Armee, starb als er acht Jahre alt war. Seine Mutter, die aus bescheidenen Verhältnissen stammte, war Nählehrerin und träumte davon, ihn frei von materiellen Sorgen als Ingenieur zu sehen. Aber als er ein Stipendium am Bukarester Militär-Lyzeum gewann, wurde seine mathematische Begabung entdeckt und gefördert. 1923 trat er in die mathematische Fakultät der Universität von Bukarest ein, drei Jahre später erhielt er seinen ersten akademischen Grad.

Im November 1929 ging er nach Paris, immatrikulierte am Statistischen Institut der Sorbonne und promovierte dort mit einer ausgezeichneten Arbeit über statistische Fluktuationen. Obwohl damals ohne jegliche Englischkenntnisse, ging Georgescu-Roegen Ende 1930 als. Stipendiat nach England, um seine Studien bei Karl Pearson in den Galton Laboratories am Universitäts-College von London fortzusetzen. Ende 1934 erhielt er ein postdoktorales Rockefeller-Stipendium an der Harvard-Universität. Dort begegnete er Joseph A. Schumpeter, der sich sofort für die mathematisch-statistischen Methoden des jungen Mannes zur Analyse von Konjunktur-Zyklen [Schumpeter arbeitete seinerzeit an dem 1939 publizierten Buch »Business Cycles«] interessierte.

\section{Georgescu-Roegens Anfang als Ökonom}

Das war der Anfang des Wirtschaftswissenschaftlers Georgescu-Roegen. Innerhalb von eineinhalb Jahren veröffentlichte er vier wirtschaftswissenschaftliche Aufsätze. Einer davon, »Marginal Utility of Money and Elasticities of Demand « (1936) war ein Urteil gegen Milton Friedman in seiner Auseinandersetzung mit A. C. Pigou. Eine weitere Abhandlung »The Pure Theory of Consumer's Behavior « (1936) deckte einzelne ganz neue Aspekte zu diesem Thema ab und wurde von Georgescu-Roegen selbst als einer seiner herausragenden Beiträge zur ökonomischen Theorie angesehen; Paul Samuelson zufolge war es tatsächlich eine bahnbrechende Entwicklung in der Wahlhandlungstheorie.

So wurde Georgescu-Roegen mit knapp dreißig Jahren bereits ein vielversprechender und umworbener Theoretiker. Harvard und Schumpeter drängten ihn zu bleiben, letzterer wollte ein theoretisch-analytisches Werk mit ihm verfassen. Es waren diesbezüglich schon Vereinbarungen für seine mögliche Rückkehr in die USA nach dem Besuch in Rumänien getroffen worden; aber ohne daß er selbst später sagen konnte warum, dankte er ab und begab sich im Sommer 1936 zurück nach Bukarest.

\section{Rückkehr nach Rumänien}

In seinem eigenen »unterentwickelten Hinterhof « ergaben sich ihm während der darauffolgenden zwölf Jahre zwei unschätzbare ökonomische Lektionen, welche sein damaliges geradezu »religiöses Vertrauen in die mathematische Ökonomie« erschütterten. Die erste wird einem Standard-Ökonomen sicherlich als Ausdruck ökonomischer Ignoranz erscheinen: Das Faktum, daß das Grenznutzenprinzip nur in einem Land der Fülle das Ensemble von Produkterstellung und gewählter Freizeit maximiert; währenddem in einem Land des Mangels (Unterentwicklung) die Menschen solange arbeiten müssen, wie sie nur irgend können, bis zum Nullpunkt der Grenzproduktivität der Arbeit. Aber selbst in entwickelten Ländern - entgegen rein neoklassischen Postulaten - werden Konsumenten nicht nur durch ein quantitatives Warenangebot gelenkt; individuelles Verhalten wird zum Beispiel auch durch die Art der Wahrnehmung von Ansprüchen beeinflußt, was nicht mit einer quantitativen Abstufung, sondern mit einer qualitativen sozialen Matrix zu tun hat.
Die zweite Lektion lieferten die Bauern Rumäniens, welche selbst in der Inflation noch Güter für Geld verkauften, ohne Rücksicht darauf, wie entwertet dieses war, weil Geld für sie immer das höchste Gut (summum bonum) war. Diese Lehren, die aus persönlicher Erfahrung und nicht aus Lehrbüchern gezogen wurden, veranlaßten ihn nicht nur, die Keynes'sche These aufzugeben, derzufolge Staatsausgaben (`geplante Inflation $<)$ das einzigartige Rezept für allgemeines Wachstum sein sollen, sondern auch von der verbreiteten Lehrmeinung abzugehen, daß der Aufschwung, insbesondere eines unterentwickelten Landes, nur durch Inflation zustande gebracht werden könne (denn dieses ist das Mittel, mit dem tatsächlich mittels ökonomischen Wachstums die privilegierten Klassen begünstigt werden). Die herkömmliche ökonomische Weisheit lieferte ihm später noch andere Gründe zum Widerspruch, insbesondere zur Standard-Produktions-Funktion. In ihr wird die wichtige Rolle natürlicher Ressourcen ignoriert - was der Öffentlichkeit erstmals im Laufe des Erdölembargos 1973/74 einsichtig wurde, wenige Jahre nachdem Georgescu-Roegen seine neue Epistemologie und den biökonomischen Ansatz entwickelt hatte.

\section{Wieder in Harvard}

Im Februar 1949 flüchtete Georgescu-Roegen mit seiner Frau, aus Furcht verhaftet zu werden; Anfang Juli kam er wieder an die.Harvard-Universität in Cambridge, Massachusetts. Dort wurde er von Wassily Leontief und E. S. Mason als Dozent und wissenschaftlicher Mitarbeiter an der ökonomischen Fakultät wiedereingesetzt. Als Professor der Nationalökonomie übersiedelte er dann an die Vanderbilt-Universität nach Nashville/Tennesse, wo er bis zum Jahr 1969, anschließend als »distinguished « Professor bis zu seinem Ruhestand 1976 lehrte.

Bald nach seiner Rückkehr nach Harvard (wo er Schumpeter noch einmal vor seinem Tod im frühen Januar $1950 \mathrm{sah}$ ), und angesichts der »wahrlich großartigen Perspektiven, die sich in der Nationalökonomie während meines langen Exils auftaten «(Georgescu-Roegen), publizierte er mehrere Aufsätze von bleibendem Wert, darunter 1954 den Klassiker über »Choice, Expectation and Mesurability«. 1951 gab er gemeinsam mit T. C. Koopmans »Activity Analysis of Production and Allocation « heraus (mit seinen späteren »flow-fund «- Darlegungen offerierte er eine radikale Alternative sowohl zur »activity analysis « wie zur Standard-Produktionsfunktion). Mit seiner Arbeit von $1960 »$ Economic Theory and 
(c) 20I0 Authors; licensee IÖW and oekom verlag. This is an article distributed under the terms of the Creative Commons Attribution Non-Commercial No Derivates License (http://creativecommons.org/licenses/by-nc-nd/3.o/), which permits unrestricted use, distribution, and reproduction in any medium, provided the original work is properly cited. 